\title{
Agresión manifiesta y ajuste psicosocial en la adolescencia
}

\section{Overt aggression and psicosocial adjustment in adolescence}

\author{
David Moreno Ruiz ${ }^{1}$, Manuel J. Ramos Corpas, Belén Martínez Ferrer \& \\ Gonzalo Musitu Ochoa. \\ Universidad Pablo de Olavide de Sevilla, España \\ (Recepción: Julio 2010 - Aceptación: Octubre 2010)
}

\begin{abstract}
Resumen
El objetivo del presente trabajo es analizar las diferencias existentes entre adolescentes con alta y baja expresión de conductas de agresión manifiesta en variables psicosociales tales como la soledad, la satisfacción vital, la actitud hacia la transgresión de las normas escolares y la actitud hacia la autoridad institucional. La muestra estaba constituida por 565 adolescentes de edades comprendidas entre los 11 y los 17 años. Los análisis estadísticos se han llevado a cabo mediante la técnica MANOVA y la prueba t. Para configurar los grupos de análisis partimos de la clasificación de la agresión manifiesta, en las dimensiones pura, reactiva e instrumental, realizada por Little, Henrich, Jones y Hawley (2003). Los datos sugirieron que los adolescentes más agresivos experimentan mayores sentimientos de soledad, menos satisfacción con sus vidas, un menor respeto a las normas escolares y muestran actitudes más negativas hacia la autoridad institucional, que los adolescentes menos agresivos. Estos resultados son coincidentes en todas las dimensiones excepto en el sentimiento de soledad, que no se encuentra en la dimensión reactiva.

Palabras claves: agresión manifiesta, soledad, satisfacción vital, actitud hacia la transgresión de las normas, actitud hacia la autoridad institucional.
\end{abstract}

\begin{abstract}
The aim of this study is to analyze the differences between adolescents with low and high overt aggression in psychosocial variables such as loneliness, life satisfaction, attitude towards breaking school rules and attitude to institutional authority. The sample was composed of 565 adolescents aged from 11 to 17 years old. Statistical analysis were conducted using MANOVAs and $\mathrm{t}$ tests. The classification of overt aggression in three dimensions: pure, reactive and instrumental, proposed by Little, Henrich, Jones and Hawley (2003), was used to establish the analysis groups. Data suggested that those adolescents showing higher levels of aggression, informed of more feelings of loneliness, lower satisfaction with their lives, less respect for school rules and a more negative attitude towards institutional authority, in comparison to less aggressive adolescents. These results are consistent in all dimensions except for the feeling of loneliness, which is not present in the reactive dimension.

Key words: overt aggression, loneliness, life satisfaction, attitude towards breaking school rules, attitude to institutional authority.
\end{abstract}

1 Correspondencia dirigida a: David Moreno Ruiz. Universidad Pablo de Olavide de Sevilla, Departamento de Ciencias Sociales, Edificio No 11, Conde de Aranda, Ctra. de Utrera, Km. 1- 41013 Sevilla (España), E-mail: dmorrui@upo.es. 


\section{Introducción}

Actualmente, el comportamiento agresivo en la escuela despierta un gran interés y preocupación en nuestra sociedad, debido a las graves consecuencias que tiene en el ajuste psicosocial de los adolescentes y en los climas escolares y familiares (Bausela, 2008; Carrillo, Ripoll-Nuñez, Cabrera \& Bastidas, 2009; Estévez, Martínez \& Musitu, 2006; Orte \& Ballester, 2007). La finalidad del presente estudio es analizar las diferencias existentes entre adolescentes con alta y baja expresión de conductas de agresión manifiesta en su ajuste psicosocial, evaluado a través de la soledad, la satisfacción vital, la actitud hacia la transgresión de las normas y la actitud hacia la autoridad formal.

Los estudios sobre la agresión diferencian este tipo de comportamiento en función de su dimensión conductual, es decir, la forma de expresarse y su dimensión intencional, es decir, la finalidad u objetivo que se pretende conseguir (Anderson \& Bushman, 2002; Ovejero, 1998; Trianes, 2000). Sin embargo, estudios más actuales y analíticos sobre la conducta agresiva, como el realizado por Little, Henrich, Jones y Hawley (2003), delimitan la agresión en torno a dos categorías principales, formas y funciones:

1. Formas o tipos de agresión. Se puede clasificar la agresión como manifiesta versus relacional. La agresión manifiesta se refiere a comportamientos que implican una confrontación directa hacia otros con la intención de causar daño (empujar, pegar, amenazar, insultar...). La agresión relacional no implica una confrontación directa entre el agresor y la víctima, y se define como aquel acto que se dirige a provocar daño en el círculo de amistades de otra persona o bien en su percepción de pertenencia a un grupo (exclusión social, rechazo social, difusión de rumores, etc.).

2. Funciones de la agresión. Se puede clasificar la agresión como defensiva (reactiva) versus ofensiva (pura e instrumental). La agresión reactiva hace referencia a comportamientos que suponen una respuesta defensiva ante alguna provocación. Esta agresión suele relacionarse con problemas de impulsividad y autocontrol, y con un sesgo en la interpretación de las relaciones sociales que se basa en la tendencia a realizar atribuciones hostiles al comportamiento de los demás. La agresión ofensiva pura hace referencia a conductas agresivas que se desencadenan sin mediar un estímulo agresivo. En su función instrumental, la agresión ofensiva, supone una anticipación de beneficios, es deliberada y está controlada por refuerzos externos. Este tipo de agresión se ha relacionado con posteriores problemas de delincuencia, pero también con altos niveles de competencia social y habilidades de líder (Carroll, Houghton, Hattie \& Durkin, 1999; Emler \& Reicher, 1995).

La operacionalización de la agresión elaborada por estos autores ha demostrado ser de gran utilidad en el estudio de la agresión escolar, debido al detallado nivel de análisis que se realiza de esta conducta (Moreno, Estévez, Murgui \& Musitu, 2009a; Musitu, Estévez \& Emler, 2007). La agresión escolar es definida, en general, como cualquier tipo de comportamiento agresivo que se da en el centro educativo, entre los que se encuentran aquéllos dirigidos a hacer daño a los alumnos, al profesorado, a objetos o material escolar y que puede ser puntual u ocasional o darse de manera repetida y mantenerse a lo largo del tiempo (Serrano \& Iborra, 2005). En esta investigación se tratará específicamente el comportamiento agresivo de tipo manifiesto en sus dimensiones reactiva, pura e instrumental.

Además, este trabajo analiza la agresión escolar desde una perspectiva psicosocial, fundamentándose su estudio en el Modelo de Desarrollo Social de Hawkins, Catalano y Miller (1992). Desde los principales supuestos de este modelo se consideran los problemas de conducta agresiva y desajustada en el adolescente como el desequilibrio entre múltiples variables. Estos agentes operan en diversos niveles de interrelación como factores de riesgo o de protección, facilitando o inhibiendo la participación en episodios problemáticos e influyendo en el desarrollo del adolescente en distintos contextos.

Por lo tanto, en el presente estudio, se analizan una serie de factores psicosociales relacionados con el ajuste en la adolescencia, que destacan tanto por su importancia en la explicación de la conducta agresiva escolar como por su novedad en el estudio de esta temática, como la soledad, la 
satisfacción con la vida, la actitud hacia la transgresión de normas sociales y la actitud hacia la autoridad institucional. De hecho, es en la actualidad cuando distintas investigaciones están mostrando la importancia de estos factores en la explicación de dicha conducta desviada (Buelga, Musitu, Murgui \& Pons, 2008; Martínez, Murgui, Musitu \& Monreal, 2009).

Diversos autores subrayan que los alumnos que no aceptan las reglas por las que se rige el funcionamiento escolar presentan una actitud más negativa hacia el profesor y la escuela, problemas de integración en el aula y mayores conductas disruptivas, que los adolescentes comprometidos con las normas del centro (Musitu et al, 2003). Del mismo modo, la soledad se relaciona con problemas de ajuste en la escuela, agresión entre iguales y bajos índices de satisfacción vital en los adolescentes (Ladd \& Troop-Gordon, 2003; Moreno, Estévez, Murgui \& Musitu, 2009b). Esta serie de vínculos, entre la actitud hacia la transgresión de las normas y figuras de autoridad escolar, la soledad y la agresión entre pares, sugiere la posibilidad de una conexión entre las experiencias de soledad de los adolescentes y su satisfacción vital.

Teniendo en cuenta estos antecedentes, el presente trabajo tiene por objeto estudiar las diferencias existentes entre adolescentes con alta y baja expresión de conductas de agresión manifiesta, en determinadas variables de ajuste psicosocial, como la soledad, la satisfacción vital, la actitud hacia la transgresión de las normas escolares y la actitud hacia la autoridad institucional. En consecuencia, la originalidad de este trabajo reside en el análisis de la conducta agresiva manifiesta evaluada a partir de sus tres dimensiones pura, reactiva e instrumental, y su relación con las variables psicosociales anteriormente enunciadas. Partimos de la siguiente hipótesis: (1) los adolescentes más agresivos tienen un mayor sentimiento de soledad, una mayor motivación hacia la transgresión de las normas, una menor satisfacción con la vida y menor respeto hacia la autoridad institucional.

\section{Método}

\section{Participantes}

Participaron en el estudio un total de 565 adolescentes españoles de ambos sexos ( $49 \%$ chicas y $51 \%$ chicos) con edades comprendidas entre los 11 y los 17 años (edad media 13.7 y desviación típica 1.4) y escolarizados en cuatro centros públicos de educación secundaria obligatoria de la provincia de Sevilla.

\section{Procedimiento}

Se seleccionaron cuatro centros de enseñanza secundaria de la provincia de Sevilla siguiendo el procedimiento de selección aleatoria. Tras obtener los permisos correspondientes de la Dirección y Consejo Escolar de cada centro, se realizó un seminario informativo con la plantilla de profesorado para explicar los objetivos, importancia y alcance del estudio en el que se solicita su compromiso de participación. Paralelamente, se envió una carta explicativa de la investigación a los padres y madres de los alumnos donde, además, se solicitó su consentimiento en relación con la participación de su hijo. Los adolescentes cumplimentaron la batería de instrumentos en sus aulas durante un periodo regular de clase de aproximadamente 45 minutos de duración. En todos los casos, la participación fue voluntaria, anónima y supervisada por investigadores previamente entrenados.

\section{Instrumentos}

Escala de Soledad UCLA de Russell, Peplau y Cutrona (1980). Esta escala adaptada por Expósito y Moya (1993) al español, se compone de 20 ítems que informan del sentimiento de soledad experimentado por el adolescente (ejemplo: "Con qué frecuencia sientes que te falta compañía"). Las respuestas van de 1 -nunca- a 4 -siempre-. El alfa de Cronbach de esta escala en la presente muestra es de .94 . 
Escala de Satisfacción con la Vida de Diener, Emmons, Larsen y Griffin (1985; traducción bidireccional inglés-español). Esta escala ofrece un índice general de satisfacción vital, entendida ésta como un constructo general de bienestar subjetivo. El instrumento consta de 5 ítems con un rango de respuesta de 1 -muy en desacuerdo- a 4 -muy de acuerdo- (ejemplo: "Mi vida es en la mayoría de los aspectos como me gustaría que fuera"). La consistencia interna medida a través del alfa de Cronbach en la presente muestra es de .81 .

Escala de Actitud hacia la Autoridad de Emler y Reicher (1995), compuesta por 20 ítems que evalúan la actitud del adolescente hacia las instituciones y figuras de autoridad formal, con un rango de respuesta de 1 -nada de acuerdo- a 4 -totalmente de acuerdo-. Esta escala mide dos dimensiones: (1) Actitud hacia la escuela y el profesorado (ejemplo: "En el colegio no me valoran como persona", "Los profesores tratan mejor a los estudiantes de clases sociales superiores"), y (2) Actitud hacia la policía, las leyes y normas socialmente establecidas (ejemplo: "La policía está para hacer una sociedad mejor para todos", "Es normal saltarse la ley si no se causa daño a nadie"). El alfa de Cronbach obtenido para estas dimensiones en la presente muestra es de .77 y .73 respectivamente.

Escala de Conducta Agresiva Manifiesta. Utilizamos para el presente estudio la subescala de agresión manifiesta de la Escala de Conducta Agresiva de Little, Henrich, Jones y Hawley (2003; traducción bidireccional inglés-español). El instrumento mide con 12 ítems y con un rango de respuesta de 1 -muy en desacuerdo- a 4 -muy de acuerdo- tres dimensiones: (1) Violencia manifiesta pura (conductas agresivas que implican agresión física o verbal sin mediar un estimulo desencadenante de la agresión; ejemplo: "Soy una persona que pega a los demás"), (2) Agresión manifiesta reactiva (conductas agresivas físicas o verbales como respuesta a la percepción de una agresión previa; ejemplo: "Cuando alguien me enfada, le hago daño o le hiero"), (3) Agresión manifiesta instrumental (conductas agresivas físicas o verbales como medio para conseguir un fin; ejemplo: "Amenazo a otros para conseguir lo que quiero"). El alfa de Cronbach obtenido para estas subescalas en la presente muestra es de $.79, .82$ y .84 respectivamente.

\section{Resultados}

Para analizar los datos de este trabajo se ha utilizado la técnica estadística del análisis multivariante de la varianza. El análisis multivariante de la varianza o MANOVA es una técnica que se utiliza para valorar la existencia de diferencias entre grupos en múltiples variables dependientes que se consideran de forma simultánea. Esta técnica informa de las diferencias entre grupos a partir de la comparación de las medias obtenidas por cada uno de ellos en las variables dependientes y permite contrastar la significación estadística de estas diferencias al considerar como hipótesis nula la igualdad de las medias comparadas.

Se realizaron tres MANOVAs con las variables dependientes soledad, satisfacción con la vida, actitud hacia la transgresión de las normas escolares y actitud hacia la autoridad institucional. Las variables independientes fueron: en el primer caso agresión manifiesta en su dimensión pura, en el segundo caso agresión manifiesta en su dimensión reactiva y en el tercer caso agresión manifiesta en su dimensión instrumental. Para poder utilizar estas variables como factores fijos, se agruparon las puntuaciones en dos niveles mediante un análisis de conglomerados, a partir de los cuales obtuvimos dos grupos de alumnos (alta agresión y baja agresión) para cada uno de los tipos de agresión estudiados: pura, reactiva e instrumental. La característica más importante del análisis de conglomerados es que se configuran grupos con la mínima variabilidad intragrupo y la máxima entregrupos.

El total de grupos examinados en este trabajo fue de 6: los grupos X1 y X2 se correspondieron respectivamente con alta y baja agresión manifiesta pura, el grupo $\mathrm{Y} 1$ se correspondió con alta agresión reactiva, el Y2 con baja agresión reactiva y los grupos Z1 y Z2 agruparon a los adolescentes con altas y bajas puntuaciones en la dimensión instrumental respectivamente. Los centroides finales de los conglomerados así como el número de participantes que conformaron cada uno de los 6 grupos elaborados se presentan en la Tabla 1. 
Tabla 1 Centroides finales y número de casos en cada conglomerado

\begin{tabular}{lcc}
\hline & \multicolumn{2}{c}{ Conglomerado } \\
\hline Centros Finales & 1 alta agresión & 2 baja agresión \\
\hline Agresión manifiesta Pura (grupo a) & 7,82 & 4,84 \\
$\mathrm{~N}^{\circ}$ Casos & 104 & 434 \\
Válidos & 538 & \\
Perdidos & 27 & \\
Agresión manifiesta Reactiva (grupo b) & 10,57 & 5,78 \\
$\mathrm{~N}^{\circ}$ Casos & 103 & 442 \\
Válidos & 545 & \\
Perdidos & 20 & 10,78 \\
Agresión manifiesta Instrumental & 5,39 & 41 \\
(grupo c) & 501 & \\
$\mathrm{~N}^{\circ}$ Casos & 542 & \\
Válidos & 23 & \\
Perdidos & & \\
\hline
\end{tabular}

Seguidamente, se efectuó un ANOVA para conocer si los grupos eran estadísticamente diferentes. Los resultados de los tres ANOVAS confirmaron que los grupos X1 y X2, Y1 y Y2 y Z1 y Z2 mostraron diferencias significativas (ver Tabla 2).

Tabla 2 Pruebas ANOVA con el conglomerado como variable independiente

\begin{tabular}{|c|c|c|c|c|c|c|}
\hline & \multicolumn{2}{|c|}{ Conglomerado } & \multicolumn{2}{|c|}{ Error } & \multirow[t]{2}{*}{$\mathrm{F}$} & \multirow[t]{2}{*}{ Sig. } \\
\hline & $\begin{array}{c}\text { Media } \\
\text { cuadrática }\end{array}$ & $\mathrm{gl}$ & $\begin{array}{c}\text { Media } \\
\text { cuadrática }\end{array}$ & $\mathrm{gl}$ & & \\
\hline $\begin{array}{l}\text { Agresión manifiesta } \\
\text { pura }\end{array}$ & 743,177 & 1 & ,786 & 536 & 944,929 &, 000 \\
\hline $\begin{array}{l}\text { Agresión manifiesta } \\
\text { reactiva }\end{array}$ & 1918,430 & 1 & 2,149 & 543 & 892,701 &, 000 \\
\hline $\begin{array}{l}\text { Agresión manifiesta } \\
\text { instrumental }\end{array}$ & 1103,181 & 1 &, 822 & 540 & 1342,689 &, 000 \\
\hline
\end{tabular}

Una vez configurados los grupos, se realizaron los MANOVAS con cada uno de los tipos de agresión como variable independiente y las variables de ajuste psicosocial seleccionadas (soledad, satisfacción vital, actitud hacia la transgresión de las normas escolares y actitud hacia la autoridad institucional). Las pruebas MANOVA resultaron significativas, como se observó a partir de sus Lambdas de Wilks (ver Tabla 3). 
Tabla 3 Pruebas MANOVA de variables psicosociales con el conglomerado como variable independiente

\begin{tabular}{lcccccc}
\hline Efecto & Valor & F & $\begin{array}{c}\text { Gl de la hipó- } \\
\text { tesis }\end{array}$ & $\begin{array}{c}\text { Gl del } \\
\text { error }\end{array}$ & $\begin{array}{c}\text { Significa- } \\
\text { ción }\end{array}$ \\
\hline $\begin{array}{l}\text { Conglomerados } \\
\text { agresión manifiesta } \\
\text { pura }\end{array}$ & $\begin{array}{c}\text { Lambda de } \\
\text { Wilks }\end{array}$ &, 563 & 13,579 & 14,000 & 245,000 &, 000 \\
$\begin{array}{l}\text { Conglomerados } \\
\begin{array}{l}\text { agresión manifiesta } \\
\text { reactiva }\end{array}\end{array}$ & $\begin{array}{c}\text { Lambda de } \\
\text { Wilks }\end{array}$ &, 702 & 7,427 & 14,000 & 245,000 &, 000 \\
$\begin{array}{l}\text { Conglomerados } \\
\text { agresión manifiesta } \\
\text { instrumental }\end{array}$ & $\begin{array}{c}\text { Lambda de } \\
\text { Wilks }\end{array}$ &, 685 & 8,038 & 14,000 & 245,000 &, 000 \\
\hline
\end{tabular}

A partir de los resultados obtenidos en los MANOVA se realizaron Pruebas $t$ para examinar entre qué variables existían diferencias significativas. En la Tabla 4 se presentan las medias y desviaciones típicas para cada uno de los grupos: grupo X1 (alta agresión pura), grupo X2 (baja agresión pura); grupo Y1 (alta agresión reactiva), grupo Y2 (baja agresión reactiva); grupo Z1 (alta agresión instrumental), grupo Z2 (baja agresión instrumental), y las variables psicosociales estudiadas, así como los resultados de la prueba $t$ de diferencia de medias.

Tabla 4. Medias, desviaciones típicas y resultados de la prueba t con los grupos (1, alta agresión; 2, baja agresión) en las tres dimensiones de agresión manifiesta

\begin{tabular}{llccc}
\hline & & Media & Media & t \\
& & 1 & 2 & \\
\hline \multirow{2}{*}{ Soledad } & Pura (grupo X) & $37.97(9.47)^{\mathrm{a}}$ & $34.98(8.45)^{\mathrm{b}}$ & $3.000^{* *}$ \\
& Reactiva (grupo Y) & $35.76(9.02)$ & $35.67(8.72)$ & $.089($ n.s.) \\
& Instrumental (grupo Z) & $39.47(9.87)^{\mathrm{a}}$ & $35.30(8.55)^{\mathrm{b}}$ & $-2.525^{*}$ \\
\hline \multirow{2}{*}{$\begin{array}{l}\text { Satisfacción } \\
\text { con la vida }\end{array}$} & Pura (grupo X) & $14.07(2.95)^{\mathrm{b}}$ & $15.97(2.91)^{\mathrm{a}}$ & $-5.755^{* * *}$ \\
& Reactiva (grupo Y) & $14.51(3.13)^{\mathrm{b}}$ & $15.83(2.93)^{\mathrm{a}}$ & $-3.732^{* * *}$ \\
& Instrumental (grupo Z) & $13.97(3.26)^{\mathrm{b}}$ & $15.78(2.95)^{\mathrm{a}}$ & $3.347^{* *}$ \\
\hline \multirow{3}{*}{$\begin{array}{l}\text { Actitud hacia } \\
\text { transgresión }\end{array}$} & Pura (grupo X) & $8.20(2.86)^{\mathrm{a}}$ & $6.09(2.38)^{\mathrm{b}}$ & $6.879^{* * *}$ \\
& Reactiva (grupo Y) & $8.01(3.06)^{\mathrm{a}}$ & $6.18(2.37)^{\mathrm{b}}$ & $6.593^{* * *}$ \\
& Instrumental (grupo Z) & $8.55(2.69)^{\mathrm{a}}$ & $6.35(2.55)^{\mathrm{b}}$ & $-4.879^{* * *}$ \\
\hline \multirow{2}{*}{$\begin{array}{l}\text { Actitud hacia } \\
\text { autoridad }\end{array}$} & Pura (grupo X) & $15.03(3.40)^{\mathrm{b}}$ & $17.17(3.31)^{\mathrm{a}}$ & $-5.783^{* * *}$ \\
& Reactiva (grupo Y) & $14.82(3.48)^{\mathrm{b}}$ & $17.17(3.35)^{\mathrm{a}}$ & $-6.116^{* * *}$ \\
& Instrumental (grupo Z) & $14.42(2.93)^{\mathrm{b}}$ & $16.92(3.43)^{\mathrm{a}}$ & $4.885^{* * *}$ \\
\hline
\end{tabular}

$\mathrm{a}>\mathrm{b} ; * \mathrm{p}<.05, * * \mathrm{p}<.01, * * * \mathrm{p}<.001$ 
La prueba t señaló que existían diferencias estadísticamente significativas en las cuatro variables individuales analizadas, en función de la dimensión agresión manifiesta pura, entre el grupo X1 y el grupo X2. Los adolescentes con mayores niveles de agresión pura mostraron puntuaciones medias más altas en soledad, que el grupo de adolescentes de baja agresión $(t=3.000, \mathrm{p}<.01)$. Sin embargo en la variable satisfacción con la vida, el grupo de adolescentes con menores niveles de agresión presentó de media puntuaciones más altas que los estudiantes que mostraron mayor agresión $(t=-5.755$, $\mathrm{p}<.001)$. Además, el grupo de adolescentes más agresivos también obtuvo medias superiores en comparación con los menos agresivos en actitud hacia la transgresión de las normas $(t=6.879, \mathrm{p}<$ $.001)$. Por el contrario, los estudiantes con baja agresión presentaron una actitud más positiva hacia las figuras de autoridad, como por ejemplo el profesor, que los adolescentes que forman parte del grupo de alumnos más agresivos $(t=-5.783, \mathrm{p}<.001)$.

En la dimensión reactiva de la conducta agresiva se encontraron diferencias en satisfacción vital, actitud hacia la transgresión y actitud hacia la autoridad entre los grupos Y1 y Y2. En la variable soledad no se encontraron diferencias entre adolescentes más agresivos y menos agresivos. En satisfacción con la vida y actitud hacia las figuras de autoridad, los alumnos que formaron parte del grupo denominado alta agresión obtuvieron puntuaciones medias más bajas en estas variables $(t=$ -3.732 y $t=-6.116, \mathrm{p}<.001$, respectivamente). Los adolescentes más agresivos mostraron una mayor actitud hacia la transgresión de las normas del centro educativo que los menos agresivos $(t=$ $6.593, \mathrm{p}<.001)$.

En la dimensión instrumental se encontraron diferencias entre los dos grupos estudiados, el Z1 y el Z2, en todas las variables individuales analizadas. Los adolescentes que utilizan de manera más frecuente este tipo de agresión puntuaron más alto en las variables soledad y actitud hacia la transgresión de las normas sociales $(t=-2.525, \mathrm{p}<.05$ y $t=-4.879, \mathrm{p}<.001$, respectivamente). Sin embargo, en las variables satisfacción con la vida y actitud hacia la autoridad formal fue el grupo de adolescentes menos agresivos el que consiguió resultados medios más elevados $(t=3.347, \mathrm{p}<.01$ y $t=4.885, \mathrm{p}<.001$, respectivamente). Por último, es necesario destacar la semejanza de los resultados que se observaron en los 6 grupos analizados en los distintos tipos de agresión.

\section{Discusión}

En el presente estudio se han analizado las diferencias entre adolescentes con alta agresión y baja agresión instrumental, en sus tres funciones pura, reactiva e instrumental, en los siguientes indicadores de ajuste psicosocial: soledad, satisfacción vital, actitud hacia la transgresión de las normas escolares y actitud hacia la autoridad institucional.

Los resultados indican que los adolescentes más agresivos en sus diferentes manifestaciones, pura, reactiva e instrumental, tienen un peor ajuste psicosocial, lo cual confirma nuestra hipótesis, aunque no en su totalidad, en el sentido de que los adolescentes más agresivos muestran una menor satisfacción con la vida, una actitud más negativa hacia la autoridad institucional y una actitud más positiva hacia la transgresión de las normas que aquéllos menos agresivos, excepto en la variable soledad, donde no se encuentran diferencias entre unos y otros en agresión manifiesta reactiva.

Una posible explicación para este resultado es que la función defensiva de la agresión está más aceptada en nuestro contexto sociocultural, lo que, a su vez, explica que sean más permisivos con sus iguales que muestran este tipo de expresiones comportamentales, al percibir que se defienden de forma legítima. Se puede inferir que estos adolescentes carecen de las habilidades sociales necesarias para solucionar estas situaciones de manera no violenta. Esta incapacidad para resolver determinados problemas de interacción con los iguales de manera no-agresiva deja entrever sentimientos de frustración, de insatisfacción con la vida, e incluso, aunque no directamente, cierto rechazo hacia las normas y figuras de autoridad formal, como es el profesor (Estévez, Murgui, Moreno \& Musitu, 2007; Martínez, Buelga \& Cava, 2007). 
Respecto de la agresión manifiesta pura, un tipo de agresión que se ejerce de manera impulsiva y sin mediar ningún estímulo aparente que lo provoque, nuestros datos sugieren que los adolescentes en los que más predomina este tipo de comportamientos tienen mayores sentimientos de soledad que sus iguales no agresivos. Este resultado converge con otros trabajos en los que se ha observado que los adolescentes que expresan este tipo de agresión son más rechazados por sus compañeros de aula, lo cual es, obviamente, un indicador del sentimiento de soledad (Cava, Musitu \& Murgui, 2007).

También podríamos explicar este comportamiento desde la subcultura de la violencia, en la medida en que hay constancia, aunque no muy fundamentada, de que en aquellos contextos o comunidades donde la violencia se asume con normalidad dentro de las relaciones familiares y sociales, niños y adolescentes expresan en sus relaciones interpersonales estos comportamientos (FernándezVillanueva, Domínguez, Revilla \& Anagnostou, 2004; Muñoz \& Navas, 2004; Wolfgang \& Ferracuti, 1982). Este es un aspecto que no se ha evaluado en este trabajo y que creemos que es de la suficiente entidad como para entender mejor las interacciones existentes entre las subculturas escolar y la cultura comunitaria. Profundizar en esta relación nos permitiría orientar con más rigor los programas y acciones sociales dirigidas a mejorar la calidad de vida (Garbarino, 1986; Garbarino, Dubrow, Kostelny \& Pardo, 1992; Guemureman, 2006).

En lo referente a la actitud hacia la transgresión de las normas sociales y la actitud hacia la autoridad institucional, se ha observado que los grupos de estudiantes más agresivos, en las tres dimensiones evaluadas, respetan menos las reglas escolares y tienen una actitud más negativa hacia las figuras de autoridad, como el profesor y la escuela, que los alumnos menos agresivos. Estos resultados confirman los obtenidos en diferentes investigaciones (Emler \& Reicher, 2005; Hoge, Andrews, \& Lescheid, 1996; Loeber, 1996; Musitu et al., 2003).

En este sentido, si se considera el modelo de la escuela tradicional, el resultado de transgredir una norma escolar implica, generalmente, un castigo impuesto por el profesor, lo cual puede conllevar para el adolescente una imagen más negativa del educador y por ende una actitud más negativa hacia su figura de autoridad. Distintos autores han observado que las experiencias del adolescente en el contexto escolar y las expectativas del profesor son dos factores muy relevantes para explicar actitudes negativas hacia el centro y profesorado (Birch \& Ladd, 1998; Molpeceres, Lucas \& Pons, 2000) y, obviamente, una actitud positiva hacia la escuela y hacia la figura del profesor puede prevenir la implicación de los estudiantes en episodios de carácter agresivo (Moncher \& Miller, 1999; Thornberry, 1996).

Creemos que una de las principales aportaciones del presente estudio, que merece por su interés ser subrayada, es la importancia de analizar la agresión desde una perspectiva multidimensional (pura, reactiva e instrumental). Esta delimitación conceptual de la agresión facilita el análisis y la comprensión del motivo por el cual el adolescente se implica en esta conducta desviada. También, cabe considerar que los resultados obtenidos contribuyen, en cierta medida, a delimitar algunas de las variables psicosociales que pueden predisponer, a ciertos adolescentes, a implicarse en incidentes agresivos en el centro educativo. Es necesario, por tanto, profundizar en su estudio con el fin de determinar sus efectos concretos tanto a corto como a largo plazo, y facilitar, de este modo, el desarrollo de programas psicoeducativos apropiados, que mejoren el ajuste psicosocial del adolescente, en particular, y el clima de convivencia, general, presente en nuestras aulas.

Por último, se debe reseñar que los resultados presentados en este trabajo deben interpretarse con cautela debido a la naturaleza transversal del estudio y correlacional de los datos, de modo que no podemos establecer relaciones causales entre las variables. Un estudio longitudinal con medidas en distintos tiempos ayudaría a la clarificación de las asociaciones aquí observadas. También sería enriquecedor obtener información de distintas fuentes (alumnos, padres y profesores) y recurrir a la triangulación metodológica, al aplicar, además de técnicas cuantitativas, alguna técnica cualitativa. 


\section{Referencias}

Anderson, C. A. \& Bushman, B. J. (2002). Human aggression. Annual Review of Psychology, 53, 27-51.

Bausela, E. (2008). Estrategias para prevenir el bullying en las aulas. Intervención Psicosocial, 17 (3), 369-370.

Birch, S. H. \& Ladd, G. W. (1998). Children's interpersonal behaviors and the teacher-child relationship. Developmental Psychology, 34, 934-946.

Buelga, S.; Musitu, G.; Murgui, S. \& Pons, J. (2008). Reputation, loneliness, satisfaction with life and aggressive behavior in adolescence. The Spanish Journal of Psychology, 11 (1), 192-200.

Carrillo, S.; Ripoll-Nuñez, K.; Cabrera, V. \& Bastidas, H. (2009). Relaciones familiares, calidad de vida y competencia social en adolescentes y jóvenes colombianos. SUMMA Psicológica UST, 6 (2), 3-18.

Carroll, A.; Houghton, S.; Hattie, J. \& Durkin, K. (1999). Adolescent reputation enhancement: differentiating delinquent, nondelinquent, and at-risk youths. Journal of Child Psychology and Psychiatry, 40, 593-606.

Cava, M. J.; Musitu, G. \& Murgui, S. (2007). Individual and social risk factors related to overt victimization in a sample of Spanish adolescents. Psychological Reports, 101, 275-290.

Diener, E. D.; Emmons, R. A.; Larsen, R. J. \& Griffin, S. (1985). The Satisfaction With Life Scale. Journal of Personality Assessment, 49, 71-75.

Emler, N. \& Reicher, S. (1995). Adolescence and delinquency. Oxford: Blackwell Pub.

Emler, N., \& Reicher, S. (2005). Delinquency: cause or consequence of social exclusion? En D. Abrams, J. Marqués \& M. Hogg (Eds.), The social psychology of inclusion and exclusion. (pp. 211-241). Philadelphia: Psychology Press.

Estévez, E.; Martínez, B. \& Musitu, G. (2006). La autoestima en adolescentes agresores y víctimas en la escuela: La perspectiva multidimensional. Intervención Psicosocial, 15 (2), 223-232.

Estévez, E.; Murgui, S.; Moreno, D. \& Musitu, G. (2007). Estilos de comunicación familiar, actitud hacia la autoridad institucional y conducta violenta del adolescente en la escuela. Psicothema, 19, 108-113.

Fernández-Villanueva, C.; Domínguez, R.; Revilla, J. C. \& Anagnostou, A. (2004). Formas de legitimación de la violencia en televisión. Política y sociedad, 41, 183-199.

Garbarino, J. (1986). Can we measure success in preventing child abuse? Issues in policy, programming and research. Child Abuse and Neglect, 10, 143-156.

Garbarino, J.; Dubrow, N.; Kostelny, K. \& Pardo, C. (1992). Children in Danger: Coping with the Consequences of Community Violence. San Francisco: Jossey-Bass.

Guemureman, S. (2006). Ni bandas ni pandillas: la miopía de las teorías de las subculturas para explicar la violencia juvenil. Revista Temas Sociológicos, 11, 153-171.

Hawkins, J. D.; Catalano, R. F. \& Miller, J. Y. (1992). Risk and protective factors for alcohol and other drug problems in adolescence and early adulthood: Implications for substance abuse prevention. Psychological Bulletin, 112, 64-105.

Hoge, R. D.; Andrews, D. A. \& Leschied, A. W. (1996). An investigation of risk and protective factors in a sample of youthful offenders. Journal of Child Psychology and Psychiatry ad Allied Disciplines, 37, 419-424.

Ladd, G. W. \& Troop-Gordon, W. (2003). The role of chronic peer difficulties in the development of children's psychological adjustment problems. Child Development, 74, 1344-1367. 
Little, T. D.; Henrich, C. C.; Jones, S. M. \& Hawley, P. H. (2003b). Disentangling the "whys" from the "whats" of aggressive behaviour. International Journal of Behavioral Development, 27, 122133.

Loeber, R. (1996). Developmental continuity, change and pathways in male juvenile problem behaviours and delinquency. En J. D. Hawkins (Ed.), Delinquency and crime: current theories (pp. 28-67). New York: Cambridge University Press.

Martínez, M.; Buelga, S. \& Cava, M. J. (2007). La satisfacción con la vida en la adolescencia y su relación con la autoestima y el ajuste escolar. Anuario de Psicología, 38 (2), 5-15.

Martínez, B.; Murgui, S.; Musitu, G. \& Monreal, M.C. (2009). El rol del apoyo parental, las actitudes hacia la escuela y la autoestima en la violencia escolar en adolescente. International Journal of Clinical and Health Psychology, 8, 679-692.

Molpeceres, M. A.; Lucas, A. \& Pons, D. (2000) Experiencia escolar y orientación hacia la autoridad institucional en la adolescencia. Revista de Psicología Social, 15, 87-105.

Moncher, F. J. \& Miller, G. E. (1999). Nondelinquent youths' stealing behavior and their perceptions of parents, school, and peers. Adolescence, 34, 577-591.

Moreno, D.; Estévez, E.; Murgui, S. \& Musitu, G. (2009a). Relación entre el clima familiar y el clima escolar: El rol de la empatía, la actitud hacia la autoridad y la conducta violenta en la adolescencia. International Journal of Psychology and Psychological Therapy, 9 (1), 123-136.

Moreno, D.; Estévez, E.; Murgui, S. \& Musitu, G. (2009b). Reputación social y violencia relacional en adolescentes: el rol de la soledad, la autoestima y la satisfacción vital. Psicothema, 21, 537542.

Muñoz, J.J. \& Navas, E. (2004). Conducta antisocial en adolescentes: teorías explicativas psicosociales. Psiquis, 25 (2), 79-86.

Musitu, G.; Estévez, E. \& Emler, N. (2007). Adjustment problems in the family and school contexts, attitude towards authority and violent behaviour at school in adolescence. Adolescence, 42, 779794.

Musitu, G.; Veiga, F. H.; Lila, M.; Martínez, B.; Herrero, J. \& Estévez, E. (2003). Conductas disruptivas e actitudes hacia la autoridad Institucional en adolescentes: el rol de la escuela. En M. F. Patrício (Ed.), Por uma Escola sem Violência: A escola Cultural. Uma resposta (pp. 649658). Porto: Porto Editora.

Orte, C. \& Ballester, L. (2007). Prevención del comportamiento delictivo en la comunidad. Intervención Psicosocial, 16 (2), 269-281.

Ovejero, A. (1998). Las relaciones humanas. Psicología social teórica y aplicada. Madrid: Biblioteca Nueva.

Russell, D.; Peplau, L. A. \& Cutrona, C. E. (1980). The revised UCLA loneliness Scale: concurrent and discriminant validity evidence. Journal of Personality and Social Psychology, 39, 472-480.

Serrano, Á. \& Iborra, I. (2005). Violencia entre compañeros en la escuela. Valencia: Centro Reina Sofía para el Estudio de la Violencia.

Thornberry, T. P. (1996). Empirical support for interactional theory: a review of the literature. En J. D. Hawkins (Ed.), Delinquency and crime: current theories (pp. 198-235). New York: Cambridge University Press.

Trianes, M. V. (2000). La violencia en contextos escolares. Málaga: Aljibe.

Wolfgang, M. E. \& Ferracutti, F. (1982). La subcultura de la violencia: hacia una teoría criminológica. México: Fondo de cultura económica. 